\title{
Differing Effects of Pulsed and CW Microwave Energy Upon Nerve Function as Detected by Birefringence Measurement
}

\author{
PETER V. K. BROWN AND LAWRENCE E. LARSEN
}

\begin{abstract}
The change in resting birefringence of crab nerve coincident with propagation of the action potential was used as a measure of peripheral nerve response to microwave radiation. Birefringence indicates membrane permeability changes associated with the ionic currents of the action potential. The use of an optical dependent variable has the advantage that no field perturbations are introduced by sensing electrodes.

Statistical analysis of the data indicated that pulsed microwave energy degraded the birefringence amplitude a greater amount and more rapidly than did either continuous wave $(\mathrm{CW})$ energy of the same average power or commensurate heating. $\mathrm{CW}$ energy and heating caused no changes from the control condition.
\end{abstract}

\section{INTRODUCTION}

$\mathrm{T}$ HE NERVOUS system is of ten considered to be especially sensitive to microwave radiation. Various studies have purported to show changes in EEG after exposure to low level pulsed microwave radiation [1]-[6]. Changes in the firing patterns of isolated neurons after stripline irradiation [7], and changes in the compound action potential of peripheral nerve at very low average power levels of microwave radiation have been reported [8].

The latter work of Kamenskiy holds interest because low average power radiation (pulsed, $0.2^{\circ} \mathrm{C}$ temperature rise, $3.5 \mu \mathrm{W} / \mathrm{cm}^{2}$ ) reportedly changed the conduction velocity by 10 percent. Chou and Guy [9] were unable to replicate Kamenskiy's results. All effects were attributable to alterations of the bathing solution temperature.

The use of glass or metal stimulating and/or receiving electrodes located in the microwave field causes artifacts and field distortion. This is due to differing microwave properties between glass or metal and the nerve bathing solution. The use of electrodes outside the field as in the case of Chou and Guy does avoid these problems, but this tactic prevents direct measurement of the irradiated portion of the nerve. Microwave transparent electrodes in the field can be used but require careful design.

Manuscript received March 11, 1980; revised June 12, 1980. The procedures used within this paper conform to standards for the use of laboratory animals established by the Institute of Laboratory Animal Resources, U.S. National Academy of Sciences (Guide for the Care and Use of Laboratory Animals, U.S. Government Printing Office, Washington, DC, DHEW Publication No. NIH 74-23).

The authors are with the Department of Microwave Research, Walter Reed Army Institute of Research, Washington, DC 20012.
The use of an optical dependent variable allows observation of the irradiated portion of the nerve without field perturbation. The optical variable used in this study is change in the resting birefringence of the nerve coincident with propagation of an action potential. The use of birefringence allows observation of action potential events in the membrane. Thus, any changes noted when the nerve is stimulated and exposed to microwave energy could be related to voltage dependent membrane permeability effects.

It has been shown that there is a change in the resting birefringence of a nerve accompanying the action potential [10]-[13]. The changes in birefringence are time coincident with the action potential and proportional to the amplitude [14]-[18]. The sources of the birefringence are thought to be longitudinally oriented proteins in the axoplasm and tangentially oriented proteins in the sheath [19], [20]. The changes in birefringence properties of a nerve are due to changes in the conformational state of the cell membrane. Cohen [18] attributes the Kerr effect, i.e., the polarization and alignment of molecules by an electric field. Another possibility is compression of the membrane dielectric in the electric field.

\section{EQUIPMENT}

The quality of the equipment used to measure birefringence is critically important; therefore, a detailed discussion of the optical components is not out of place. See Fig. 1. The light source was a $250-\mathrm{W}$ tungsten halogen lamp with a flat element (Leitz \#37723) installed in a lamp housing (Leitz 514-309) and powered from a regulated 25-V 10-A power supply (HP 6268A). The light was passed through a heat filter (Leitz BG23) and then was projected into a Leitz Trinocular Ortholux microscope body. The light was then changed from a horizontal to vertical path by a mirror and then passed through the first polarizer (Polaroid HN32 $\times 0.030^{\prime \prime}, 1 \times 10^{-4}$ extinction ratio, transmission 25 percent). The polarized and attenuated light then entered the condenser lens (Leitz 512081; condenser base A. 0.25) and then the cap lens (Leitz 513-437; A 0.45/L20). The light beam exited the cap lens and entered the WR 284 waveguide and was focused on the specimen nerve. 


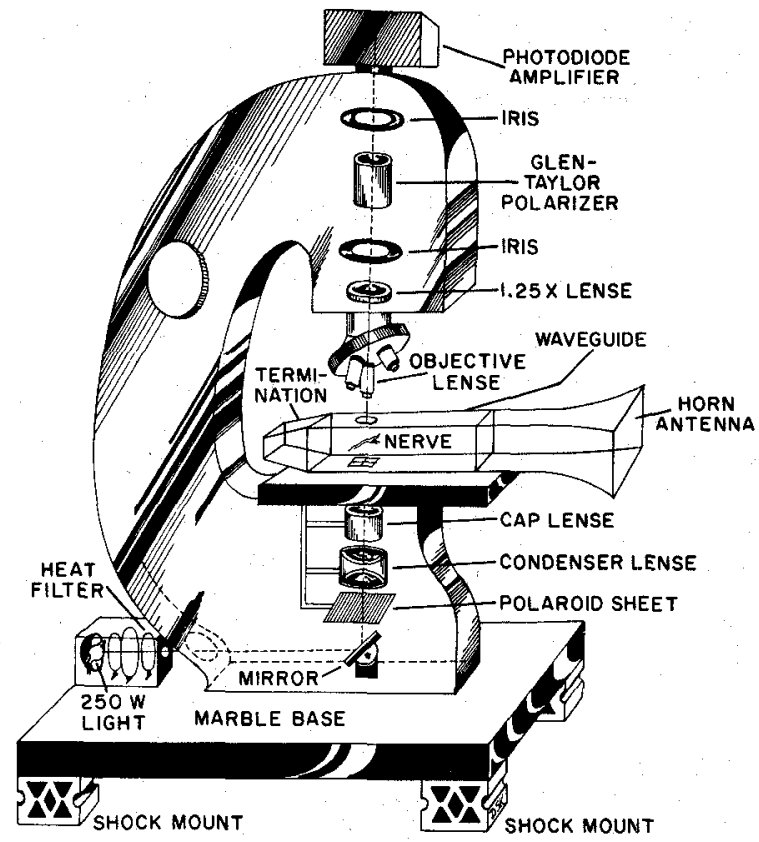

Fig. 1. Optical path for birefringence measurement including terminated waveguide with receiving horn for microwave energy exposure of nerve. Nerve holder not shown.

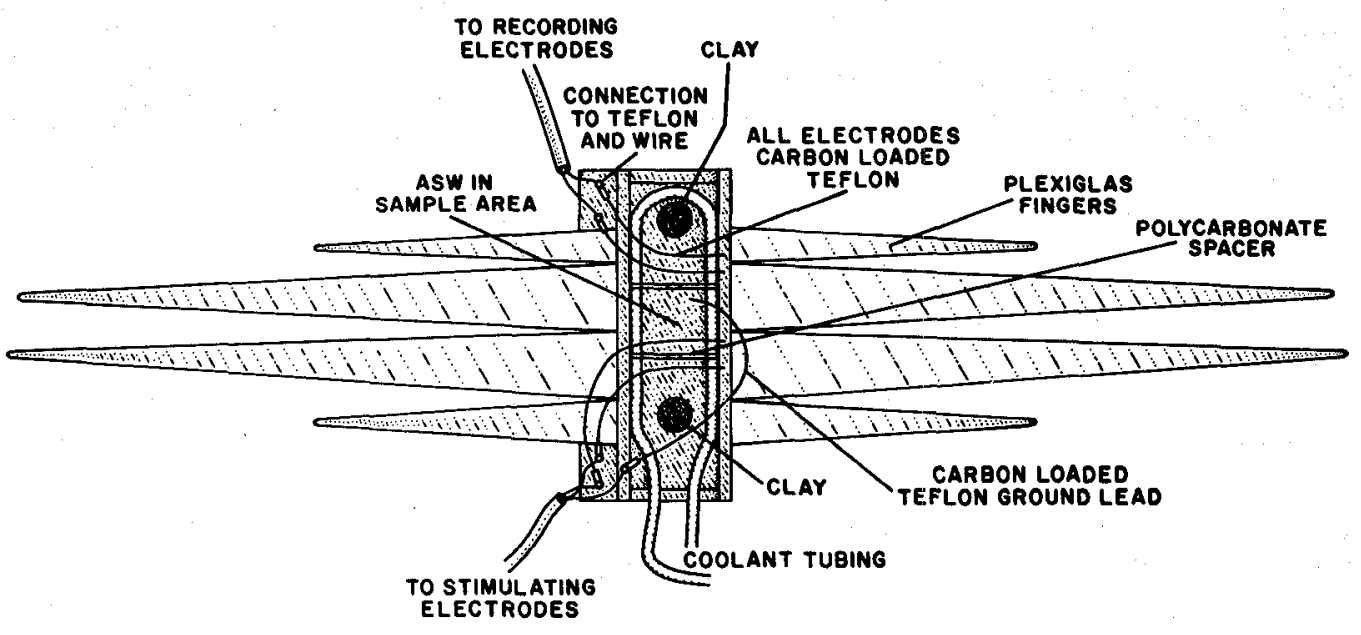

Fig. 2. Top view of nerve chamber.

The light that passed through the nerve was received by the objective lens (Leitz 559-005; special objective UM $32 \times / 0.30$, free working distance $1.40 \mathrm{~mm}$, not strain free) mounted external to the waveguide. Inside the microscope tube, the light beam passed through a $1.25 \times$ lens, then an iris, then the second polarizer (Karl Lambrecht MGT-YA20, Glean-Taylor type, calcite, " $A$ " grade, extinction ratio $5 \times 10^{5}: 1$, transmission 45 percent), then a second iris. The two iris were needed to restrict the angle of incidence and emergence from the calcite polarizer. This second polarizer was set at $90^{\circ}$ to the first one, and $45^{\circ}$ to the long axis of the specimen nerve.

The light emitted from the second polarizer (when a nerve was present) was converted by a large photodiode (EG \& G Inc., UV444B photovoltaic diode, $1 \mathrm{~cm}^{2}$, sensitivity $0.6 \mathrm{~A} / \mathrm{W}, \mathrm{NEP} 2.5 \times 10^{-14} \mathrm{~W} / \sqrt{\mathrm{Hz}}$ ). The output current of the photodiode was processed by a current to voltage converter/amplifier (gain $2.5 \times 10^{9} \mathrm{~V} / \mathrm{A}$, output noise with diode connected $50 \mathrm{mV}$ peak to peak; passband $100 \mathrm{~Hz}$ to $5 \mathrm{kHz}$ ). The light source and microscope were place on a $89-\mathrm{cm} \times 61-\mathrm{cm} \times 8.5-\mathrm{cm}$ thick marble slab, which was supported by four damped rubber shock mourts (Lord Kinematics \# J-5984-1) with a 0.25-Hz, 3-dB cutoff frequency.

The nerve chamber (Fig. 2) was an open top rectangular box $1.3 \mathrm{~cm} \times 3.5 \mathrm{~cm} \times 1 \mathrm{~cm}$ deep. Thin notched spacers of polycarbonate defined a sample area $1.3 \mathrm{~cm} \times 1.3 \mathrm{~cm} x$ $0.5 \mathrm{~cm}$ deep. This was filled with artificial sea water (ASW) during the experiment. Both the stimulating and recording electrodes were made of medium resistance $(1000 \Omega / \mathrm{cm})$ carbon loaded Teflon filament. Connections to flexible copper wires were made near the sidewalls of 


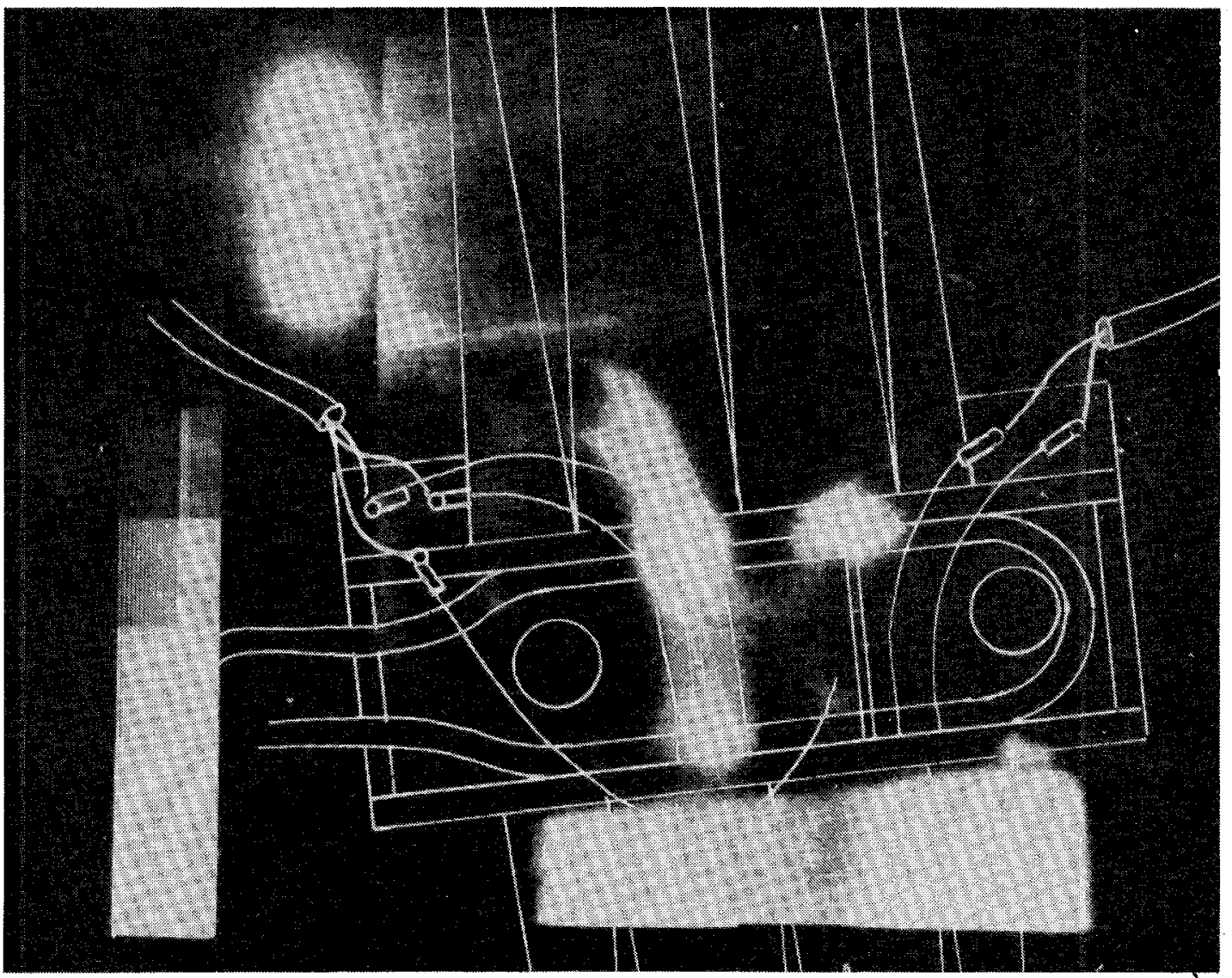

Fig. 3. Thermograph of nerve chamber filled with saline gelatine mixture. Overlay of nerve chamber shows uniform heating in nerve area; hot spots where carbon loaded Teflon leads attach to plexiglas, or are in free air. Quantized in $0.38^{\circ} \mathrm{C}$ steps. Total heating approximately $3.5^{\circ} \mathrm{C}$.

the waveguide where the $E$ field approaches zero. The recording electrodes were on the other side of one polycarbonate spacer while one stimulating electrode was in the ASW and the other on the far side of the second polycarbonate spacer. A ground lead of medium resistance carbon loaded Teflon filament was immersed in the ASW. The exterior stimulating and receiving wires were firmly attached to the marble block, and then connected to external stimulator (Nuclear Chicago 7150) and differential amplifier $(G=1000$, noise $8 \mathrm{nV} / \sqrt{\mathrm{Hz}}$, bandwidth $1 \mathrm{kHz}$ ).

To prevent radiation of microwave energy into the environment and maintain a high effective coupling, a waveguide exposure system was used (see Fig. 1). A 47-cm section of WR 284 waveguide was mounted on the microscope stage. A $1.3-\mathrm{cm}$ round hole in the upper, broad side allowed observation of the nerve, and a $2.3-\mathrm{cm}$ square hole (with screening dividing it into four $1.2-\mathrm{cm}$ square holes) allowed entrance of light from the condenser lens assembly at the lower broad side of the waveguide. Four $0.5-\mathrm{cm}$ holes in the middle of the narrow sides of the waveguide allowed access for coolant circulating hoses and stimulus-recording.

Long triangular plexiglas pieces were attached to the nerve chamber. These pieces extended parallel to the direction of energy propagation and provided dielectric impedance matching and a uniform field in the sample area. Uniform field induction in the specimen vicinity where optical measurements are made was verified by infrared thermography (Fig. 3). The nerve and its exposure apparatus were centered under the microscope objective lens. The waveguide section was terminated (Narda 304C, $10 \mathrm{~W}$ avg) on one end and attached to an antenna (Narda 644) on the other. This entire assembly was mounted on the microscope stage, and thus was isolated from vibration due to the heavy marble microscope pedestal.

Approximately $0.15 \mathrm{~cm}$ from the antenna was an identical one, mounted on a pedestal that was not vibration isolated. This antenna was supplied either with $\mathrm{CW}$ microwave energy (General Radio 1360-B oscillator, Alfred 5020 amplifier) or pulsed microwave energy (Applied Microwave Laboratory model PG5KB source with 5238H/B8 head). Power was measured with standard directional couplers and power meters (Pacific Measurements Peak Power Meter 1018, HP 432A power meter, HP 779-D and 797-D couplers, HP 8491A attenuators). The coupling efficiency of this arrangement was approximately 90 percent; the remainder being lost in impedance mismatch and dissipative losses. 


\section{Procedure}

The experimental specimen was the large upper leg nerve of a common blue crab (Calinectinaes). The crabs were obtained locally and kept in an aquarium filled with artificial sea water (ASW) (specific gravity (SG) 1.022, $p \mathrm{H}$ 7.7-8.4, $T=21^{\circ} \mathrm{C}$ ). Care was taken to monitor the $p \mathrm{H}$ of the ASW frequently as the birefringence amplitude was strongly dependent on $p \mathrm{H}$. Crabs were kept a minimum of overnight; some were kept for 2 months before use. If the crabs survived overnight, they would last indefinitely.

In order to be sure that the observed effects were due to microwave energy and not physiological variations of nerve condition, nerves were compared from the same animal, in pairs. Two legs were cut off the specimen crab at the same time to provide a matched pair. The nerves were immediately dissected from the legs and placed in ASW (3-5 min). After letting the nerves soak for another few minutes, the first nerve dissected out was used in the experiment. After the nerve was run in either radiated or sham irradiated conditions, the second nerve was used. The delay from first to second nerve was about $30 \mathrm{~min}$ after dissection. Each experiment took about 23 $\min /$ nerve.

The ligated nerve was stretched across the electrodes and the threads secured in small lumps of dielectric (clay) at the end of the chamber. The nerve was mounted perpendicular to the direction of microwave propagation and parallel to the broad face of the waveguide with its long axis at $45^{\circ}$ to the polarization of the incident light. The sample area had been filled with ASW, as well as each end area. Cellophane tape placed over the end area formed a miniature cloud chamber with saturated air keeping the nerve ends moist. The optical path intersected the nerve near the stimulating electrodes. The nerve at this point was completely immersed in the ASW. The nerve was observed close to the stimulation electrode because the birefringence signal became weaker and less sharp at greater distances due to dispersion of conduction velocities of the axons in the compound action potential.

During the experiment, the nerve was stimulated once every $1.8 \mathrm{~s}$. The current from the stimulator was typically $10 \mathrm{~mA}$; however, a great deal of this was shorted out by residual electrolyte between the medium resistance lines. The stimulus level chosen was $2 \mathrm{~mA}$ above that which gave maximum birefringence; or $10 \mathrm{~mA}$. The same stimulus current was used for both nerves, i.e., the level was set using the first nerve of the pair.

One nerve of the pair was exposed to microwave energy. When run in "order 1", the first nerve was exposed to microwave energy and the second nerve was the control, not exposed to microwave energy. When run in "order 2", the control, nonexposed nerve came first with the second nerve exposed to microwave energy.

Nerves were exposed to either pulsed microwave energy (5-kW peak $1-\mu$ s pulsewidth 1000 -pps, $5-W$ average input power to apparatus) or $\mathrm{CW}$ microwave energy (5-W aver- age input power to apparatus), or to no energy (control). As measured by temperature rise of the nerve's bathing solution with the cooler off, the specific absorption rate was $123 \mathrm{~mW} / \mathrm{g}$.

The same paired leg procedure was used in a control experiment where heated electrolyte simulated the microwave energy condition. In this case, the first nerve was tested with heated electrolyte and the second nerve was tested with cold electrolyte as in the control condition for the other experiments. The temperature of the coolant was set either to give a temperature rise in the nerve chamber equivalent to that produced by microwave energy, or set at the usual control value. The change in resting birefringence of the nerve was observed as the nerve was stimulated. This signal was recorded on one track of an F]M tape recorder (HP 3955 system) along with the stimulus and resulting action potential.

Whereas the nerve was constantly stimulated during the experiment, AP and birefringence data were recorded only during minutes $0-2,7-9,14-16$, and $21-23$ of the experiment. Later the birefringence signal from the tape was processed by a signal averager (Fabri Tek Inc. model 1052LS). See Fig. 4. A 1024-point sample, $50 \mu \mathrm{s}$ per address, 64 sweep average was used. The averaged signal was then plotted (HP-Moseley 2D-2A) and the peak amplitude measured. Separate averaging and analysis were made for each time interval.

Data on each nerve of the pair from the same crab was first normalized with respect to the first measurement on each nerve. Variation in nerve size, fiber composition and stimulus thresholds made the comparison of the absolute amplitudes not meaningful. The analytical appropriateness of the normalization procedures (i.e., covariance compensation/correction) was verified with bivariate plots that confirmed linearity and origin intersection.

The data was then analyzed using three different nonparametric tests of high power efficiency. They were chosen because they minimized both type I and type III statistical errors. Type I errors express the probability of incorrectly rejecting the null hypothesis. This has been set at $p=0.05$ for the analysis. Type I errors occur due to misapplication of the test statistic in its critical region. Type II errors express the probability of incorrectly accepting the null hypothesis, i.e., missing a significant difference. They occur due to inefficient use of the data in the probability calculations. Parametric tests such as the $t$-test are not properly applied to the data because the distributional assumptions are violated. This leads to incorrect probabilities for a type I error, and increases the probabilities of a type II error. The tests selected have weak or no distributional assumptions and a high power efficiency. The test with the highest power efficiency has the smallest probability of a type II error.

The statistical tests used were the Randomization Test for matched pairs (power efficiency $(\mathrm{PE})=100$ percent), the Walsh Test $(P E=99$ percent) and the Wilcoxon 


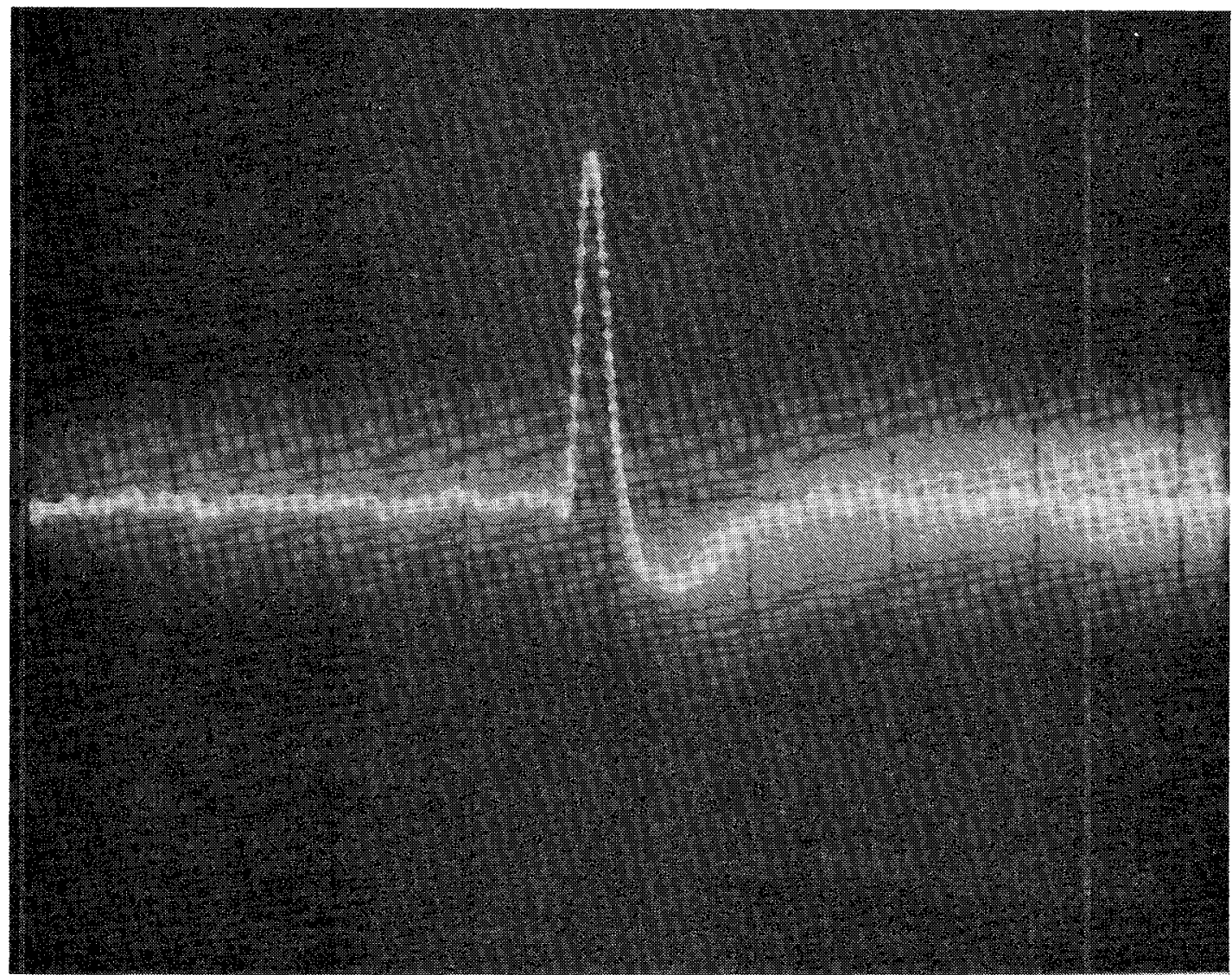

Fig. 4. Typical birefringence signal after signal averaging $(64 \times)$. Amplitude was measured from baseline to peak. Horizontal axis $4 \mathrm{~ms} / \mathrm{cm}$, vertical axis arbitrary voltage units.

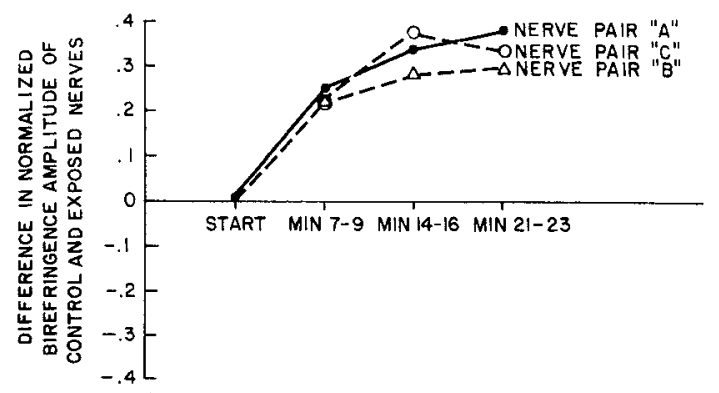

Fig. 5. Typical data for 3 nerves exposed to microwave energy; a positive difference in amplitude means that control nerve had larger normalized birefringence amplitude.

Matched-Pairs Signed-Ranks Test ( $\mathrm{PE}=95$ percent) [2226]. The null hypothesis $H_{0}$ was tested at a 0.05 probability threshold. $H_{0}$ may be stated that the birefringence amplitude (after normalization) of the control nerve is the same as the birefringence amplitude (after normalization) of the paired exposed nerve, at each time interval. In addition, the slopes of the best fit (least squares method) straight line through differences of the amplitude of control and exposed nerve pairs over time were calculated. The slopes for various exposure conditions were com-

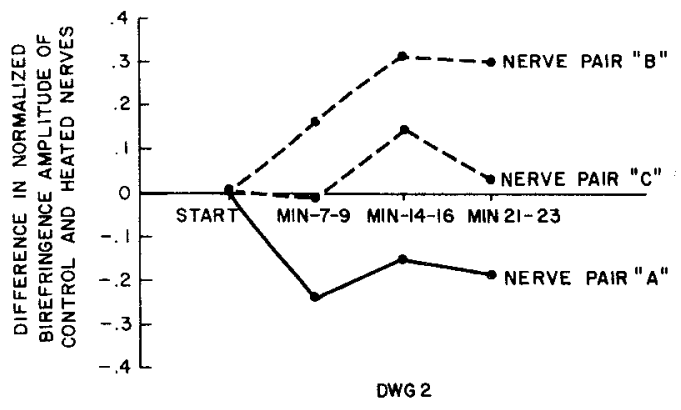

Fig. 6. Typical data for 3 nerves exposed to equivalent heating caused by absorption of microwave energy, a negative difference in amplitudes means that heated nerve had larger normalized birefringence amplitude.

pared and analyzed using the Pitman approximation to the Randomization Test for Independent Samples. In this case, the null hypothesis may be stated that the rate of degradation under the various exposure conditions is identical.

\section{Results}

The results of the data analysis are given for the normalized birefringence amplitude at each time interval (Figs. 5 and 6). Unless otherwise noted, the test result 
applies to all time intervals:

\begin{tabular}{|c|c|c|c|}
\hline Condition & $\begin{array}{l}\text { Randomization Test for } \\
\text { Matched Pairs (PE } 100 \text { percent) }\end{array}$ & $\begin{array}{l}\text { Walsh Test } \\
\text { (PE } 99 \text { percent) }\end{array}$ & $\begin{array}{l}\text { Wilcoxon Matched- } \\
\text { Pairs Signed-Rank } \\
\text { Test (PE } 95 \text { percent) }\end{array}$ \\
\hline $\begin{array}{l}\text { Pulsed } \mu \text {, order } 1 \\
\text { Pulsed } \mu \text {, order } 2\end{array}$ & $\begin{array}{l}\text { Rejected, } P=0.002 \\
\text { Rejected, } P=0.03\end{array}$ & $\begin{array}{l}\text { Rejected, } P=0.01 \\
\text { Rejected time } 3 \\
P=0.05 \\
\text { Accepted time } \\
1,2\end{array}$ & $\begin{array}{l}\text { Rejected, } P=0.01 \\
\text { Rejected time } 3 \\
P=0.05 \\
\text { Accepted time } \\
1,2\end{array}$ \\
\hline \multirow[t]{2}{*}{$\mathrm{CW} \mu$, order 1} & $\begin{array}{l}\text { Rejected time } 1 \\
P=0.05\end{array}$ & - & - \\
\hline & Accepted time 2, 3 & Accepted & Accepted \\
\hline $\mathrm{CW} \mu$, order 2 & Accepted & Accepted & Accepted \\
\hline Hot water exposure & Accepted & Accepted & Accepted \\
\hline
\end{tabular}

Data for the electrical action potential was recorded and is summarized below:

\begin{tabular}{lllll}
\hline Condition & $\Delta T$ & AP Amplitude & $\begin{array}{l}\text { Change Conduction } \\
\text { Velocity (Control) }\end{array}$ & $\begin{array}{l}\text { Change in Conduction } \\
\text { Velocity (Exposed) }\end{array}$ \\
\hline Pulsed $\mu$,order 1 & $3^{\circ} \mathrm{C}$ & No change & -10 percent approx. & +10 percent approx. \\
Pulsed $\mu$, order 2 & $3^{\circ} \mathrm{C}$ & No change & -12 percent approx. & +12 percent approx. \\
$\mathrm{CW} \mu$, order 1 & $3^{\circ} \mathrm{C}$ & No change & -10 percent approx. & +10 percent approx. \\
$\mathrm{CW} \mu$, order 2 & $3^{\circ} \mathrm{C}$ & No change & -8 percent approx. & +16 percent approx. \\
Hot water & $4^{\circ} \mathrm{C}$ & - & - & - \\
\hline
\end{tabular}

Statistical comparison of the slopes of the differences in birefringence amplitudes, between exposure conditions is given below. The test results refer to the $H_{0}$ that the slopes are the same for the indicated comparison:

\begin{tabular}{|c|c|c|c|c|}
\hline- & Pulse, Order 1 & Pulse, Order 2 & $\mathrm{CW}$, Order 1 & $\mathrm{CW}$, Order 2 \\
\hline Pulse, order 2 & Accepted & - & - & - \\
\hline $\mathrm{CW}$, order 1 & $\begin{array}{l}\text { Rejected, } \\
P<0.001\end{array}$ & $\begin{array}{l}\text { Rejected, } \\
0.01<p<0.02\end{array}$ & - & - \\
\hline $\mathrm{CW}$, order 2 & Accepted & Accepted & $\begin{array}{l}\text { Rejected, } \\
0.02<p<0.05\end{array}$ & - \\
\hline $\begin{array}{l}\text { Hot water } \\
\text { Exposure }\end{array}$ & $\begin{array}{l}\text { Rejected, } \\
0.001<p<0.01\end{array}$ & Accepted & Accepted & Accepted \\
\hline
\end{tabular}

\section{Discussion}

The data is noisy, but the three statistical tests substantially agree. The Randomization Test (highest power efficiency) is most likely to be correct, while the Wilcoxon Test is the weakest and more prone to type II errors than the others.

All tests show a significant difference in birefringence amplitude caused by pulsed microwave exposure, when the exposure is done first. When the exposed nerve is second (order 2) the statistical tests do not agree, but the strongest test indicates there is an effect. This order effect is puzzling, since when the nerves were run in order 1 , with the control nerve last, the experiment was biased against showing a decrease in birefringence amplitude, due to the specimen degradation. Presumably, the control nerve could degenerate during the $23 \mathrm{~min}$ the first nerve was being tested.

There appears to be a very weak order effect between $\mathrm{CW}$ order 1 and $\mathrm{CW}$ order 2 exposure, with the Randomization Test showing a difference in amplitude in one time period only. Otherwise, the data show that $\mathrm{CW}$ energy exposure does not cause a significant change in birefringence amplitude relative to the control nerve.
All tests show no significant difference in birefringence amplitude to be caused by simple heating (hot water exposure). The heating simulated the long term heating of the nerves that took place with microwave exposure. The action potential data, measured outside the field, show no amplitude change. Thus, the birefringence changes were not sufficient to stop conduction of the nerve. However, observation of a compound action potential resulting from many fibers with different thresholds complicates the interpretation. Changes in conduction velocity are those expected for the heating.

The slope of the differences in birefringence amplitude between control and exposed nerves over time indicates the differential rate of degradation of the birefringence amplitudes, i.e., whether the exposed nerve birefringence is decreasing at a faster rate than the control nerve birefringence. By comparing the slopes under various exposure conditions one can discriminate exposure conditions.

The time rate of change in birefringence with pulse order 1 exposure is different from $\mathrm{CW}$ order 1 and from hot water exposure. An order sensitivity is present, with 
TEST I

Normalized Birefringence Amplttudes, Pulsed Microwave EXPOSURE

\begin{tabular}{|c|c|c|c|c|c|c|}
\hline \multicolumn{7}{|c|}{ Pulsed Energy Exposure; Order 1 (first nerve exposed, second nerve control) } \\
\hline \multicolumn{3}{|c|}{ EXPOSED } & \multicolumn{4}{|c|}{ CONTROL } \\
\hline NERVE 1.0. & TIME 2 & TIME 3 & TIME 4 & TIME 2 & TIME 3 & TIME 4 \\
\hline A & 0.60 & 0.40 & 0.30 & 0.85 & 0.73 & 0.67 \\
\hline 8 & 0.92 & 0.92 & 0.83 & 1.10 & 1.10 & 1.10 \\
\hline c & 0.55 & 0.34 & 0.21 & 0.79 & 0.62 & 0.50 \\
\hline D & 0.63 & 0.83 & 0.51 & 0.93 & 0.93 & 1.00 \\
\hline$E$ & 0.74 & 0.63 & 0.56 & 0.97 & 1.00 & 0.89 \\
\hline $\mathrm{F}$ & 0.97 & 0.59 & 0.59 & 1.14 & 1.00 & 1.10 \\
\hline G & 0.77 & 0.76 & 0.59 & 1.08 & 1.08 & 1.00 \\
\hline $\mathrm{H}$ & 0.75 & 0.66 & 0.60 & 1.05 & 0.79 & 1.07 \\
\hline I & 0.55 & 0.15 & 0.09 & 0.67 & 0.50 & 0.37 \\
\hline J & 0.80 & 0.66 & 0.52 & 0.74 & 0.74 & 0.59 \\
\hline k & 0.85 & 0.50 & 0.35 & 0.67 & 0.47 & 0.33 \\
\hline$L$ & 0.72 & 0.64 & 0.52 & 0.75 & 0.57 & 0.50 \\
\hline M & 0.69 & 0.54 & 0.42 & 0.74 & 0.56 & 0.48 \\
\hline \multicolumn{3}{|c|}{ Pulsed Energy Exposure; Or } & 2 (second & nerve exposed, & , first $n$ & (erve control) \\
\hline NERVE I.D. & $\begin{array}{l}\text { CONTROL } \\
\text { TIME } 2\end{array}$ & TIME 3 & TIME 4 & $\begin{array}{l}\text { EXPOSED } \\
\text { TIME } 2\end{array}$ & TIME 3 & TIME 4 \\
\hline A & 0.75 & 0.61 & 0.54 & 0.71 & 0.43 & 0.29 \\
\hline B & 1.0 & 0.81 & 0.81 & 0.95 & 0.82 & 0.82 \\
\hline c & 0.70 & 0.55 & 0.45 & 0.83 & 0.73 & 0.73 \\
\hline 0 & 1.0 & 1.0 & 0.93 & 1.0 & 0.83 & 0.83 \\
\hline E & 1.44 & 1.44 & 1.44 & 0.89 & 0.74 & 0.63 \\
\hline$F$ & 0.78 & 0.69 & 0.66 & 0.67 & 0.48 & 0.37 \\
\hline G & 1.29 & 1.21 & 1.07 & 1.15 & 1.0 & 0.92 \\
\hline H & 0.85 & 0.85 & 0.78 & 0.77 & 0.63 & 0.53 \\
\hline I & 1.17 & 1.17 & 1.22 & 1.04 & 1.04 & 0.96 \\
\hline
\end{tabular}

TEST II

Normalized Birefringence Amplitudes, CW Microwave EXPOSURE

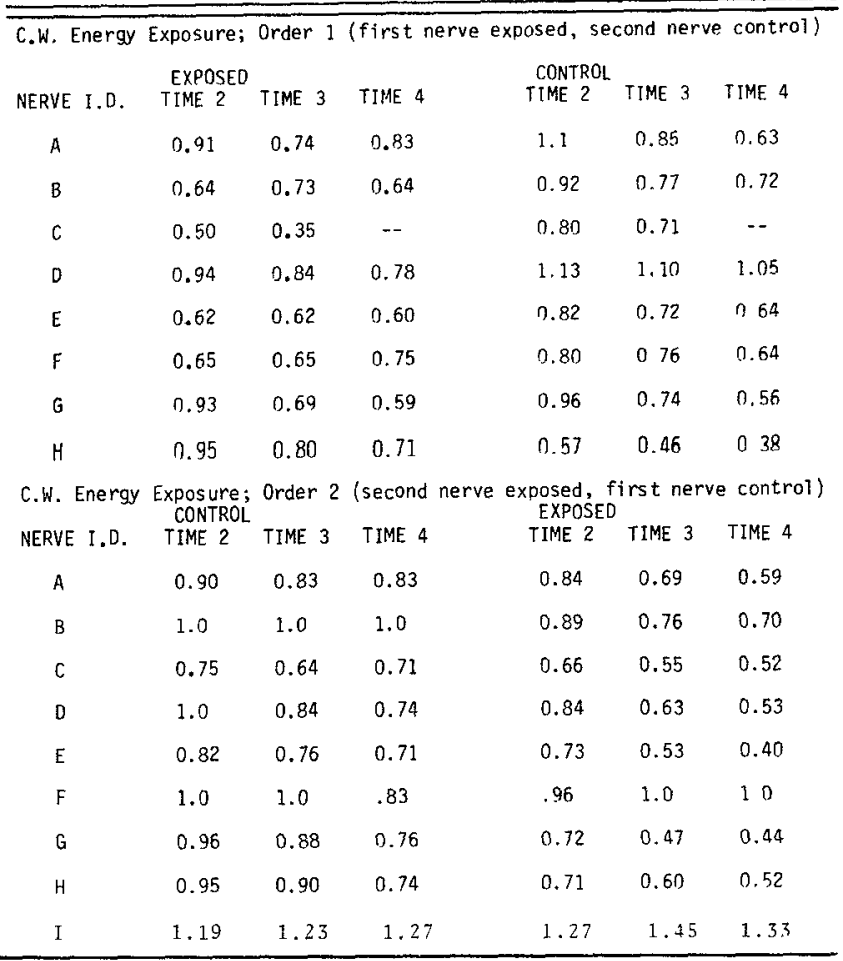

TEST III

\begin{tabular}{|c|c|c|c|c|c|c|}
\hline \multirow{2}{*}{\multicolumn{7}{|c|}{ Heat Energy Exposure (first nerve exposed, second nerve control) }} \\
\hline & EXPOSED & & & CONTRO & & \\
\hline NERVE I.D. & TIME 2 & TIME 3 & TIME 4 & TIME 2 & TIME 3 & TIME 4 \\
\hline A & 0.92 & 0.75 & 0.75 & 0.68 & 0.60 & 0.56 \\
\hline B & 0.70 & 0.46 & 0.39 & 0.98 & 0.77 & 0.72 \\
\hline$c$ & 1.0 & 0.87 & 0.83 & 0.87 & 0.83 & 0.57 \\
\hline D & 0.96 & 0.68 & 0.60 & 0.95 & 0.83 & 0.63 \\
\hline E & 0.76 & 0.59 & 0.59 & 0.83 & 0.78 & 0.72 \\
\hline $\mathrm{F}$ & 0.65 & 0.55 & 0.50 & 0.85 & 0.65 & 0.59 \\
\hline G & 0.84 & 0.76 & 0.76 & 0.94 & 0.79 & 0.66 \\
\hline H & 0.78 & 0.59 & 0.52 & 0.94 & 0.90 & 0.82 \\
\hline I & 0.96 & 0.83 & 0.74 & 0.72 & 0.56 & 0.48 \\
\hline
\end{tabular}

pulse order 2 exposure having a different effect than $\mathrm{CW}$ order 1, but puzzlingly, no difference from hot water exposure. CW order 1 exposure is different from $\mathrm{CW}$ order 2 exposure, but both are similar in degradation rate to hot water exposure.

The Pitman Test is subject to inaccuracies from kurtosis (third moment). If kurtosis is unduly large, then the accuracies of the probabilities of a type I error place some limitations upon the interpretation.

These conclusions differ from those of Chou and Guy in that the birefringence effects were not attributable to temperature changes. However, the action potential data support their conclusions to the extent that differences in action potential amplitude and velocity between control and exposed nerve were produced and attributable to bathing solution temperature changes.

Kamenskiy reported a "nonthermal" $\left(0.2^{\circ} \mathrm{C}\right)$ conduction velocity change of 10 percent with pulsed microwave energy exposure. The data show a 10-percent velocity change with a $2^{\circ} \mathrm{C}$ heating; Chou and Guy report the same data. These data are inconsistent with Kamenskiy's work.

However, the present experiment is not directed toward these parameters which have known temperature sensitivity [21]. Rather, the design minimizes temperature effects and examines a parameter related to conformational states of the membrane. Unlike action potential amplitude or conduction velocity, the nerve birefringence amplitude does not track the temperature change of the nerve, which is in agreement with Cohen [18].

\section{CONClusion}

Pulsed microwave energy affects nerve function more than continuous wave energy of the same average power. The rate of degradation of birefringence amplitude is greater for pulsed energy than CW energy. Heating the nerve gives results similar to the continuous wave energy exposure. These results are based upon the reduction over time in the peak amplitude of the fast part of the AP induced change in resting birefringence of the nerve. No other change in the shape of the birefringence signal was 
evident, but this could reflect the low resolution of the optical system.

\section{ACKNOWLEDGMENT}

Thanks are given to Lawrence Cohen of Yale University for his assistance and advice with respect to measurement of birefringence, to Leonard Binstock of NIH for advice on the maintenance and use of myxicola, to Michael Vrabec for establishing a marine holding facility that has served myxicola infundibulum, logio pealei, lolligumula, and calinectinaes, and to Leon Butler for his expert technical assistance in many aspects of the project especially with respect to specimen preparation, dissection procedures, and consistant execution of the experimental protocol.

\section{REFERENCES}

[1] C. K. Chou and A. W. Guy, "The effects of electromagnetic fields on the nervous system," Technical Report, Scientific Report \#6, Bioelectromagnetics Research Lab., Dep. of Rehabilitation Medicine, Univ. of Washington School of Medicine, Seattle, WA, Tech. Rep.; Scientific Rep. 6, Aug. 1975.

[2] J. H. Heller, "Biological effects and health implications of microwave radiation," U.S. Dept. Health, Education and Welfare, Rockville, MD, Rep. BRH/DBE 70-2 (PB193 858), 1970, pp. 116-121.

[3] R. A. Chizhenkova, "The role of different brain formations in electroencephalographic responses of rabbits to a constant magnetic field and to ultrahigh and superhigh electromagnetic fields," Zh. Vysshey Nerunoy Deyatel'Nosti, vol. 17, no. 2, pp. 313-321, 1967.

[4] R. A. Chizhenkova, "Brain biopotentials in the rabbit during exposure to electromagnetic fields," Fiz. Zh. SSSR, vol. 53, no. 5, pp. 514-519, 1967.

[5] N. N. Livshits, "The role of the nervous system in reactions to UHF fields," Biofizika, no. 3, p. 372, 1957.

[6] A. S. Pressman, Electromagnetic Fields and Life. New York: Plenum, 1970, pp. 156- 158, pp. 125- 132.

[7] S. R. Wachtel and W. Joines, "Effects of low intensity microwaves on isolated neurons," Ann. N.Y. Acad. Sci. vol. 247, pp. 46-62, 1975.

[8] Y. I. Kamenskiy, "The influence of microwaves on the functional condition of the nerve," Biofizika, vol. 9, no. 6, pp. 695-700, 1964.

[9] C. K. Chou and A. W. Guy, "Effects of Electromagnetic Fields on
Isolated Nerve and Muscle Preparations," IEEE Trans. Microwave Theory Tech., vol. MTT-26, no. 3, pp. 141-147, Mar. 1978.

[10] L. B. Cohen, R. D. Keynes, and E. B. Hille, "Light scattering and birefringence changes during nerve activity," Nature, vol. 218, pp. 438-441, May 4, 1968.

[11] A. Watanabe and S. Terakawa, "Initial and delayed birefringence signals and membrane potential of a crayfish giant axon," Proc. Japan Acad, vol. 50, no. 1, pp. 90-95, 1974.

[12] A. Watanabe, S. Terakawa, M. Nagano, "Axoplasmic Origin of the Birefringence Change Associated with Excitation of a Crab Nerve," Proc. Japan Acad., vol. 49, no. 6, pp. 470-475, 1973.

[13] W. J. Adelman, Jr., Biophysics and Physiology of Excitable Membranes. New York: Van Nostrand Reinhold, 1971, pp. 247-262.

[14] W. N. Ross, "Changes in absorption, fluorescence, dichroism, and birefringence in stained giant axons: optical measurement of mentbrane potential," J. Membrane Bio., vol. 33, pp. 141-155, 1977.

[15] J. P. Reuben, ElectroBiology of Nerve, Synapse, and Muscle. New York: Raven, 1976, pp. 13-26.

[16] I. Tasaki and K. Sisco, "Electrophysiological and optical methods for studying the excitability of the nerve membrane," Methods Membrane Bio. vol. 5, pp. 163-194, 1975.

[17] I. Tasaki, A. Watanabe, R. Sandlin, and L. Carnay, "Changes in fluorescence, turbidity, and birefringence associated with nerve excitation," Proc. Nat. Acad. Sci. U.S., vol. 61, pp. 883-888, 1968.

[18] L. B. Cohen, B. Hille, R. D. Keynes, D. Landowne, and E. Rojas, "Analysis of the potential-dependent changes in optical retardation in the squid giant axon," J. Physiol., vol. 218, pp. 205-237, 1971.

[19] F. O. Schmitt and R. S. Bear, "The optical properties of vertebrate nerve axons as related to fiber size," $J$. Cellular Comparative Physiol., vol. 9, pp. 261-273, 1937.

[20] R. S. Bear and F. O. Schmitt, "The optics of nerve myelin," J. Opt. Soc. Amer., vol. 26, pp. 206-212, 1936.

[21] W. J. Adelman, Jr., Biophysics and the Physiology of Excitable Membranes. New York: Van Nostrand Reinhold 1971, pp. 320323.

[22] H. Scheffe, "Statistical inference in the non-parametric case," Ann. Math. Stat., vol. 14, pp. 305-332, 1943.

[23] R. A. Fisher, Statistical Methods for Research Workers. Edinburgh: Oliver and Boyd, 1934.

[24] J. E. Walsh, "Applications of some significance tests for the median which are valid under very general conditions," J. Amer. Stat. Assoc., vol. 44, pp. 342-355, 1949.

[25] F. Wilcoxon, "Probability tables for individual comparison by ranking method," Biometrics, vol. 3, pp. 119-122, 1949.

[26] E. J. G. Pitman, "Significance tests which may be applied to samples from any populations," Supplement J. Royal Stat. Soc., vol. 4, pp. 119-130, 1937. 